\title{
Pedaleando por un ahorro
}

\section{Make savings by pedaling}

GÓMEZ-VILLALOBOS, Elizabeth*†, MARTÍNEZ-VALDIVIESO, Lizeth Aline, CANTORALMORENO Sheyla y SOSA-ZARATE, Jaime

\section{Universidad Politécnica de Chiapas}

ID $1^{\mathrm{er}}$ Autor: Gómez-Villalobos, Elizabeth / ORC ID: 0000-0002-4580-7065, CVU CONACYT ID: 972970

ID $1^{\text {er }}$ Coautor: Martínez-Valdivieso, Lizeth Aline / ORC ID: 0000-0001-8677-0361, CVU CONACYT ID: 973100

ID $2^{\text {do }}$ Coautor: Cantoral-Moreno, Sheyla / ORC ID: 0000-0001-9899-3853, CVU CONACYT ID: 973097

ID $3^{\text {er }}$ Coautor: Sosa-Zarate, Jaime / ORC ID: 0000-0001-8075-1899, CVU CONACYT ID: 1000680

\section{Resumen}

En este proyecto se presenta el diseño de un sistema de generación de energía eléctrica, que aprovecha el movimiento de una persona al ejercitarse sobre una bicicleta estática. Dicho sistema se implementa en un gimnasio ubicado en Suchiapa, Chiapas. Se realiza el análisis de tres categorías de personas: deportista de alto rendimiento, deportista ocasional y persona sedentaria, para evaluar las calorías consumidas por día y tiempo de ejercicio en bicicleta estática. Con esta información se obtiene un estimado de la energía que se genera en un día, para comparar el recibo de consumo eléctrico de CFE antes y después del sistema. La bicicleta estática se sujeta mecánicamente a un generador mediante un soporte, seguido por un diodo que permite el paso de la corriente eléctrica con características similares a un interruptor, el cual se conecta a un regulador de voltaje que mantiene un nivel de tensión constante. El regulador se acopla al acumulador eléctrico y a un inversor. Este último se encarga de hacer la conversión del voltaje de entrada de corriente directa y salida de corriente alterna. Al implementar el mecanismo con nueve bicicletas estáticas de generación de electricidad, se obtiene un ahorro energético del $17.57 \%$.

\section{Energía cinética, Calorías, Ahorro energético}

\begin{abstract}
This project presents the design of an electrical power generation system, which takes advantage of a person's movement when exercising on a static bicycle. This system is implemented in a gym located in Suchiapa, Chiapas. The analysis is carried out of three categories of people: high performance athlete, occasional athlete and sedentary person, to evaluate the calories consumed per day and exercise time on static bicycle. This information provides an estimate of the energy generated in one day, to compare the receipt of power consumption of CFE before and after the system. The static bicycle is mechanically attached to a generator by means of a support, followed by a diode allowing the passage of the electric current with characteristics similar to a switch, which is connected to a voltage regulator that maintains a constant voltage level. The regulator is coupled to the electric accumulator and an inverter. The latter is in charge of converting the direct current input voltage and alternating current output. By implementing the mechanism with nine static electric bicycles, an energy saving of $17.57 \%$ is obtained.
\end{abstract}

Kinetic energy, Calories, Energy saving

Citación: GÓMEZ-VILLALOBOS, Elizabeth, MARTÍNEZ-VALDIVIESO, Lizeth Aline, CANTORAL-MORENO Sheyla y SOSA-ZARATE, Jaime. Pedaleando por un ahorro. Revista de Ingeniería Eléctrica. 2019. 3-11: 1-6

\footnotetext{
* Correspondencia del Autor (alinemtzv98@ gmail.com)

$\dagger$ Investigador contribuyendo como primer autor.
} 


\section{Introducción}

La característica fundamental de la energía es que puede ser aprovechada según sea su necesidad. La electricidad se ha convertido en un elemento indispensable en la vida del ser humano, gracias a esta el hombre vive con varias comodidades. La energía eléctrica que se genera puede ser usada de forma inmediata o esta puede ser almacenada en baterías para luego ser utilizada. Este mecanismo es muy simple y de fácil operación, y lo que lo hace aún más atractivo es que garantiza generación, mientras las piezas estén funcionales, el mantenimiento es accesible y es de bajo costo.

En este documento se presenta el diseño de una aplicación energética basada en el aprovechamiento de la energía cinética desarrollada por un individuo sobre una bicicleta de spinning. Generalmente, esta energía es transformada y desperdiciada en forma de calor, resultante de la fricción entre la transmisión, el volante y el freno de estas unidades. Optamos por incurrir en estos tipos de sitios donde la actividad física sea el principal tema, beneficiaría a que la salud de las personas cambie positivamente, gracias a que es más fácil quemar calorías en bicicleta, y está energía ejercida para adelgazar o mejora la salud sería utilizada para generación de electricidad.

\section{Consideraciones}

\section{Distribución de calorías en el cuerpo humano}

La cantidad de energía que se gasta es variable y resulta de la suma de diferentes necesidades calóricas obligatorias (metabolismo basal) y otras que dependen de cada estilo de vida y de la actividad física que se desarrolle.

Las recomendaciones de la Organización Mundial de la Salud - (OMS) establecen un aporte calórico de 2000 a $2500 \mathrm{Kcal} /$ día para un varón adulto y de 1500 a $2000 \mathrm{kcal} /$ día para las mujeres.

Estas necesidades disminuyen a medida que las personas se hacen mayores. Un hombre de 65 años de constitución media necesitará unas 1900-2100 kcal/día, mientras que una mujer de 65 años de constitución media oscilará entre 1500 - $1700 \mathrm{kcal} /$ día. [1]
La Asociación Americana de Dietética (American Dietetic Association) ofrece la siguiente fórmula para poder calcular cuántas calorías necesita cada día para mantener su peso al mismo nivel:

Descubrir el índice de metabolismo basal tomando su peso actual y multiplicándolo por 10 si es mujer, por 11 si es hombre.

Añadir un $20 \%$ a este número si se es sedentario (si no se hace ejercicio físico); un $30 \%$ si se es un poco activo (camina, juega golf); un $40 \%$ si se hace ejercicio moderado (camina rápido, corre un poco, levanta pesas 31 ligeras o hace bicicleta), y un $50 \%$ si se es muy activo (correr, squash, levanta pesas, ciclismo $\mathrm{y}$ natación).

Sumar $10 \%$ al total (para digestión y absorción de nutrientes) para obtener su total de calorías diarias permitidas. [1]

\section{Parámetros del gimnasio "Extrava gym"}

Se debe tener como consideración que la siguiente información fue suministrada por el profesional encargado y dueño del gimnasio “Extrava gym”, Diego Vázquez. En la tabla 1 se muestra la información que nos sirve para conocer el campo de implementación del mecanismo propuesto.

\begin{tabular}{|l|l|}
\hline \multicolumn{2}{|c|}{ Datos generales } \\
$\begin{array}{l}\text { Horario de funcionamiento del } \\
\text { gimnasio }\end{array}$ & $\begin{array}{l}8: 00 \text { am }-1: 00 \mathrm{pm} ; \\
4: 00 \mathrm{pm}-10: 00 \mathrm{pm}\end{array}$ \\
\hline Bicicletas disponibles & 9 bicicletas \\
\hline $\begin{array}{l}\text { Personas que asisten al } \\
\text { gimnasio diariamente }\end{array}$ & 60 personas \\
\hline $\begin{array}{l}\text { Personas que utilizan la } \\
\text { bicicleta en un día }\end{array}$ & 25 personas \\
\hline $\begin{array}{l}\text { Tiempo aproximado de uso de } \\
\text { las bicicletas por día }\end{array}$ & $7 \mathrm{~h}$ \\
\hline
\end{tabular}

Tabla 1 Información general del gimnasio Extrava gym Fuente: Elaboración Propia

\section{Parámetros generales}

Se tomó en cuenta ciertos parámetros establecidos para la correcta realización de los cálculos, como son los siguientes [2]:

$\begin{array}{ll}- & 1 \text { caloría } \rightarrow 4,1868 \mathrm{~J} \\ - & 1 \mathrm{~W} \rightarrow 1 \mathrm{~J} / \mathrm{s} \\ - & 1 \mathrm{~min} \rightarrow 60 \mathrm{~s}\end{array}$

GÓMEZ-VILLALOBOS, Elizabeth, MARTÍNEZVALDIVIESO, Lizeth Aline, CANTORAL-MORENO Sheyla y SOSA-ZARATE, Jaime. Pedaleando por un ahorro. Revista de Ingeniería Eléctrica. 2019 
Otro factor importante es conocer la forma en que se distribuye las calorías, de modo que se podrá conocer con precisión cuantas calorías pueden utilizar un individuo para el ejercicio, que al final son las calorías que nos ayudarán a producir energía eléctrica.

Distribución de calorías en el cuerpo:

1. Metabolismo basal: $60 \%$

2. Actividad física: $30 \%$

3. Digestión de los alimentos y absorción de nutrientes: $10 \%$

\section{Comparación de características}

Se evaluaron tres casos de personas con diferentes características para enmarcar las diferencias y el estado de calorías del cuerpo.

\section{Deportista de alto rendimiento}

Se tienen como datos evaluativos los suministrados por el entrenador profesional, dueño del gimnasio. En la tabla 2 se muestran las características que cumple un deportista de alto rendimiento.

\begin{tabular}{|l|r|}
\hline \multicolumn{2}{|c|}{ Características } \\
\hline Energía consumida por día & $5,000 \mathrm{cal}$. \\
\hline Tiempo de ejercicio en bicicleta estática & $60 \mathrm{~min}$. \\
\hline Tiempo total de ejercicio & $240 \mathrm{~min}$. \\
\hline
\end{tabular}

Tabla 2 Especificaciones de un deportista de alto rendimiento

Fuente: Elaboración Propia

Se plantea la siguiente regla de tres, para así saber la energía obtenida por el nivel de calorías consumidas.

1 caloría $\rightarrow 4,1868 \mathrm{~J}$

5,000 Calorías $\rightarrow$ Energía generada por día

La energía generada en un día de acuerdo a un consumo de 5,000 calorías es de 20, 934 J. A partir de la energía generada en una hora, se realiza la estimación en relación con la energía generada por un día.

$60 \mathrm{~W}=\frac{(20,934 \mathrm{~J})(60 \mathrm{~min} .)}{3,600 \mathrm{~s}}$

$60 \mathrm{~W}=348.9 \frac{\mathrm{J}}{\mathrm{s}}$
Se considera la siguiente regla de tres para obtener las calorías que el cuerpo puede invertir en actividad física según el metabolismo y las calorías consumidas.

$$
\begin{aligned}
& 5000 \text { calorías } \rightarrow 100 \% \\
& \text { Actividad física } \rightarrow 30 \%
\end{aligned}
$$

De ello obtenemos que para la actividad física se disponen de 1500 calorías. Posteriormente se calcula las calorías gastadas por día, realizando ejercicio.

Calorías gast $=$

1500 Calorías - $\left(348.9 \frac{\mathrm{J}}{\mathrm{s}}\right)(4 \mathrm{~h})$

Calorías gast $=104.4$ Calorías

\section{Deportista ocasional}

En la tabla 3 se muestran los datos que cumple un individuo que practica ejercicio de manera ocasional.

\begin{tabular}{|l|c|}
\hline \multicolumn{2}{|c|}{ Características } \\
\hline Energía consumida por día & $2,500 \mathrm{cal}$. \\
\hline Tiempo de ejercicio en bicicleta estática & $30 \mathrm{~min}$. \\
\hline Tiempo total de ejercicio & $90 \mathrm{~min}$. \\
\hline
\end{tabular}

Tabla 3 Especificaciones de un deportista ocasional Fuente: Elaboración Propia

Se muestra la siguiente regla de tres, para así saber la energía obtenida por el nivel de calorías consumidas.

1 caloría $\rightarrow 4,1868 \mathrm{~J}$

2,500 calorías $\rightarrow$ Energía generada por día

La energía generada por día con un consumo de 2,500 calorías es de 10,467 J.

$$
\begin{aligned}
& 30 \mathrm{~W}=\frac{(10,467 \mathrm{~J})(30 \mathrm{~min})}{1,800 \mathrm{~s}} \\
& 30 \mathrm{~W}=174.45 \frac{\mathrm{J}}{\mathrm{s}}
\end{aligned}
$$

Se realiza la siguiente regla de tres para obtener las calorías que el cuerpo puede invertir en actividad física según el metabolismo y las calorías consumidas.

2,500 Calorías $\rightarrow 100 \%$
Actividad física $\rightarrow 30 \%$

GÓMEZ-VILLALOBOS, Elizabeth, MARTÍNEZVALDIVIESO, Lizeth Aline, CANTORAL-MORENO Sheyla y SOSA-ZARATE, Jaime. Pedaleando por un ahorro. Revista de Ingeniería Eléctrica. 2019 
Para la actividad física se disponen de 750 calorías. Calorías gastadas por día, realizando ejercicio:

\section{Calorías gast}

$=750$ Calorías $-\left(174.45 \frac{\mathrm{J}}{\mathrm{s}}\right)(3 \mathrm{~h})$

Calorías gast $=226.65$ Calorías

\section{Persona sedentaria}

En la tabla 4 se muestran los datos específicos que cumple un individuo que no practica ejercicio.

\begin{tabular}{|l|c|}
\hline \multicolumn{2}{|c|}{ Características } \\
\hline Energía consumidas por día & $3,000 \mathrm{cal}$ \\
\hline Tiempo de ejercicio en bicicleta estática & $10 \mathrm{~min}$. \\
\hline Tiempo total de ejercicio & $20 \mathrm{~min}$. \\
\hline
\end{tabular}

Tabla 4 Especificaciones de una persona sedentaria Fuente: Elaboración Propia

Del mismo modo se lleva a cabo la siguiente regla de tres, para así saber la energía obtenida por el nivel de Calorías.

\section{1 calorías $\rightarrow 4,1868 \mathrm{~J}$ \\ 3,000 calorías $\rightarrow$ Energía generada por día}

La energía que genera un individuo sedentario en un día es de 12,560.4 J

$10 \mathrm{~W}=\frac{(12,560.4 \mathrm{~J})(10 \mathrm{~min})}{600 \mathrm{~s}}$

$10 \mathrm{~W}=209.34 \mathrm{~J} / \mathrm{s}$

Se plantea la siguiente regla de tres para obtener las Calorías que el cuerpo puede invertir en actividad física según el metabolismo y las calorías consumidas.

\section{3,000 Calorías $\quad \rightarrow 100 \%$ \\ Actividad física $\rightarrow 30 \%$}

De esta operación se obtiene que para la realización de actividad física se disponen de 900 calorías.

Calorías gastadas por día, realizando ejercicio: Calorías gast $=$

900 Calorías - $\left(209.34 \frac{\mathrm{J}}{\mathrm{s}}\right)(2 \mathrm{~h}$

Calorías a gastar $=481.32$ Calorías

\section{Materiales}

Los materiales por utilizar para la construcción del sistema son: motor de 24-36 v, un controlador de $24 \mathrm{v}-12 \mathrm{v}$ y 20 A, batería, perfiles, abarcones, tornillo y arandelas para la construcción de la base y cables de calibre 12 para realizar las conexiones necesarias.

\section{Modelo del mecanismo}

El aprovechamiento de energía se dará al convertir la energía cinética en energía eléctrica, a partir de un generador de corriente continua. Este tipo de generador puede operar para un amplio margen de velocidades, tal como es el régimen de uso de una bicicleta estática. En la bicicleta se sujetará mecánicamente un generador por medio de una estructura de soporte. Posteriormente irá conectado un regulador de voltaje para realizar la función de mantener un nivel de tensión constante, dicho regulador irá enlazado a un acumulador eléctrico (batería) con el fin de almacenar la energía para ser devuelta más tarde en casi toda su totalidad. También el regulador estará de la mano con el inversor, el cual se encargará de hacer la conversión del voltaje de entrada de Corriente Directa (DC) en otro voltaje de salida de Corriente Alterna (AC). Por último esta energía será entregada para abastecer al gimnasio de energía eléctrica. Este proceso se muestra en la imagen 1.

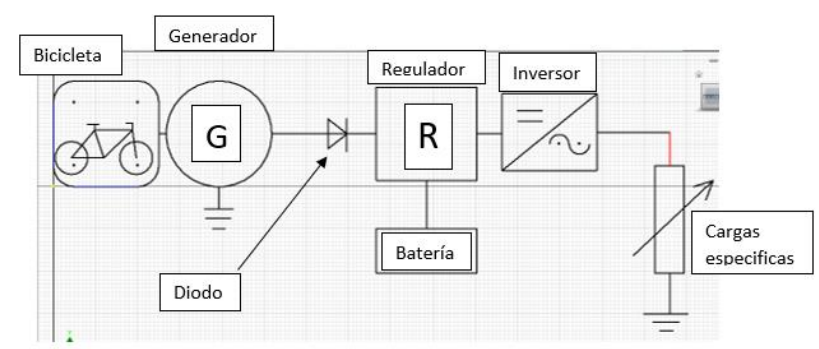

Figura 1 Diagrama del prototipo para el proyecto "Gimnasio sustentable"

Fuente: Elaboración Propia

\section{Construcción}

Para aprovechar el giro de la llanta en la producción de corriente continua, se colocó un generador, el cual acopla el rodillo de este a la llanta de la bicicleta estática, el cuerpo del generador se fijó a la estructura metálica de la bicicleta para evitar que la inercia de la llanta lo mueva de lugar. Como se muestra en la imagen 2.

GÓMEZ-VILLALOBOS, Elizabeth, MARTÍNEZVALDIVIESO, Lizeth Aline, CANTORAL-MORENO Sheyla y SOSA-ZARATE, Jaime. Pedaleando por un ahorro. Revista de Ingeniería Eléctrica. 2019 


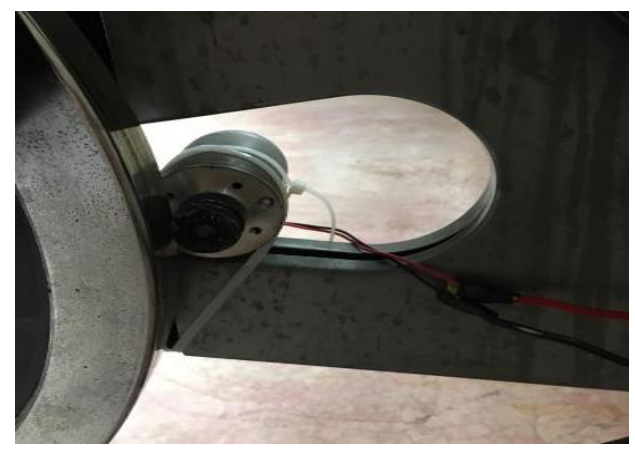

Figura 2 Generador de 24v-36v Fuente: Elaboración Propia

El generador y la batería de $12 \mathrm{~V}$ se conectan al controlador de carga, como se muestra en la imagen 3. Esta conexión se realiza con el fin de proteger a la batería al no permitir una sobrecarga o descarga en la misma. De acuerdo a la norma CFE-L0000-15-1992 se especificaron los colores de los conductores eléctricos quedando como negativo el color negro y positivo el color rojo.

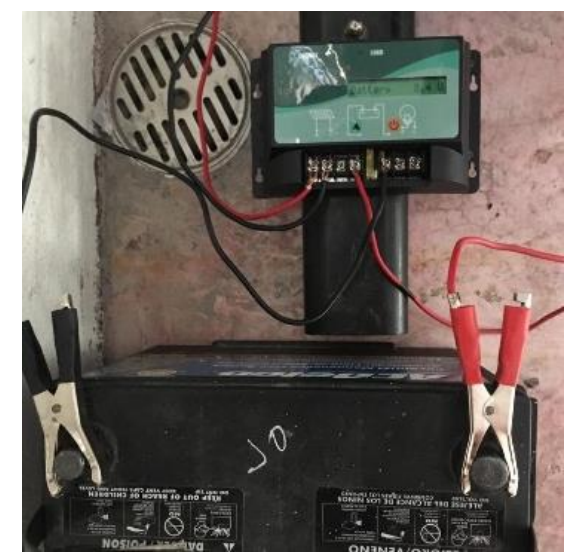

Figura 3 Conexión del sistema generador de electricidad Fuente: Elaboración Propia

\section{Metodología}

Se conoce que la demanda de energía en un bimestre para el gimnasio es de $217 \mathrm{kWh}$, como se muestra en el anexo A del recibo de consumo eléctrico de CFE, de la sección de anexos. Según el análisis del recibo, se tiene que generar alrededor de $8 \mathrm{kWh}$ en un día. Una vez se tienen los datos del gimnasio y la relación de generación se puede determinar el valor de la energía producida en un día y un bimestre. Tomando como referencia el voltaje del motor, en este caso $24 \mathrm{~V}$ y la intensidad de $1.2 \mathrm{~A}$.
Se calculó la potencia teórica para después conocer la energía almacenada en la batería en un rango de $7 \mathrm{~h}$, ya que es el tiempo estimado de uso en un día, como se muestra en la siguiente expresión matemática.

$\mathrm{P}=(\mathrm{V})(\mathrm{I})$

$\mathrm{P}=(24 \mathrm{v})(1.2 \mathrm{~A})=28.8 \mathrm{~W}$

$\mathrm{E}_{\text {almacenada }}=(28.8 \mathrm{~W})(7 \mathrm{~h})=201.6 \mathrm{Wh}$

De acuerdo a la clasificación implementada en el estudio previo y a las fórmulas 9 y 10, un deportista de alto rendimiento alcanza un voltaje de $17 \mathrm{~V}$ y una potencia de $20.4 \mathrm{~W}$ logrando almacenar una energía de 142.8 Wh, El deportista ocasional obtuvo un voltaje de $12 \mathrm{~V}$ y una potencia de 14.4 W con ello logró almacenar $100.8 \mathrm{Wh}$ y finalmente una persona sedentaria llega a producir $6.5 \mathrm{~V}$ como se muestra en la imagen 4. Por lo tanto, logró una potencia de $7.8 \mathrm{~W}$ y almacena una energía de $54.6 \mathrm{Wh}$.

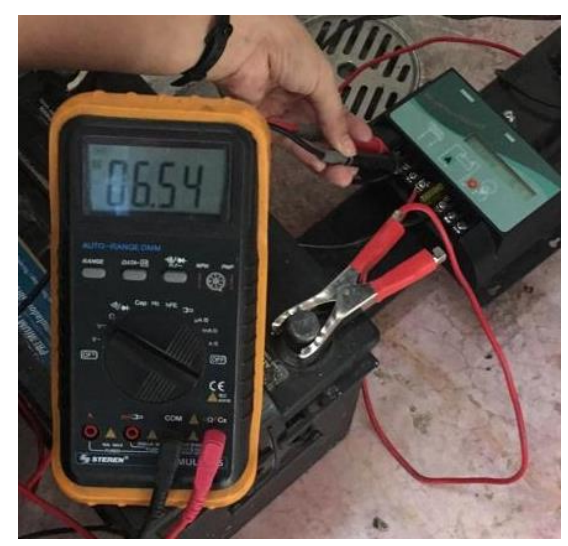

Figura 4 Voltaje obtenido por una persona sedentaria Fuente: Elaboración Propia

\section{Resultados}

\section{Comparación de la energía generada}

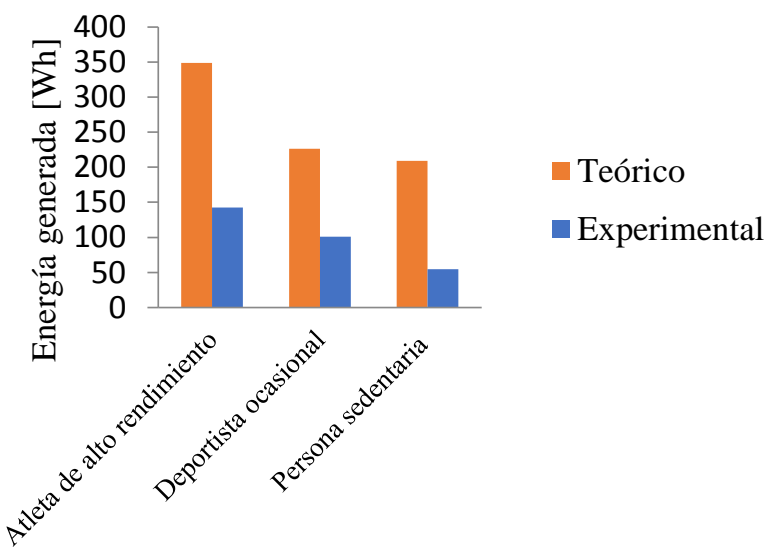

Gráfico 1 Comparación de la energía generada de datos experimentales y teóricos

Fuente: Elaboración Propia 
El ahorro energético máximo alcanzado por este mecanismo se obtiene a partir de la siguiente expresión.

$A_{E}=\left(E_{\text {almacenada }}\right)($ No. Bicicletas $)(60$ días $) d_{i} h_{p}$

$\mathrm{A}_{\mathrm{E}}=(142.8 \mathrm{Wh})(9)(60$ días $)(0.866)(0.571)$

$\mathrm{A}_{\mathrm{E}}=38.130 \mathrm{kWh}$

Donde la energía almacenada es la que produce un deportista de alto rendimiento, los 60 días es el periodo de facturación del recibo de CFE, $d_{i}$ es el factor de corrección por los días del bimestre inhábiles y $h_{p}$ es el factor de corrección del tiempo perdido por el relevo de personas en la bicicleta. Por lo tanto el porcentaje de ahorro energético equivale a:

$$
\begin{aligned}
& 217 \mathrm{kWh} \rightarrow 100 \% \\
& 38.13 \mathrm{kWh} \rightarrow \mathbf{1 7 . 5 7} \%
\end{aligned}
$$

\section{Anexos}

\begin{tabular}{|l|l|}
\hline Periodo & Energia (kWh) \\
del 13 SEP 18 al 14 NOV 18 & 217 \\
del 13 JUL 18 al 13 SEP 18 & 216 \\
del 15 MAY 18 al 13 JUL 18 & 215 \\
del 13 MAP 18 al 15 MAY 18 & 83 \\
del 12 ENE 18 al 13 MAP 18 & 63 \\
del 14 NOV 17 al 12 ENE 18 & 31 \\
del 13 SEP 17 al 14 NOV 17 & 83 \\
ded 13 III 17 al 13 SEP 17 & 14 \\
\hline
\end{tabular}

Anexo A. Recibo de CFE proporcionado por el ginmasio "Extrava gym", Fuente: Extrava gym.

\section{Agradecimiento}

Se agradece a la Universidad Politécnica de Chiapas y al gimnasio Extrava gym por el apoyo para la realización de este proyecto.

\section{Conclusiones}

$\mathrm{Al}$ implementar este mecanismo de generación de electricidad, el gimnasio lograría abastecer alrededor del $17.57 \%$ de su demanda energética. Debido a la simplicidad del sistema y los grandes beneficios que este brinda, se hace viable tener este mecanismo para suplir parte de la energía demandada dado que este sistema logra aprovechar la energía que se emplea para quemar grasa en la generación de electricidad, generando un ahorro económico para el lugar y fomentando una vida sana que ayuda al planeta.
El uso del proyecto pedaleando por un ahorro tiene como plan contrarrestar la demanda eléctrica, cabe mencionar que el estudio realizado fue bajo condiciones del gimnasio Extravagym ubicado en Suchiapa, Chiapas.

\section{Sugerencias}

Si se deseara la autosuficiencia se requeriría de dos aspectos, aumentar el número de bicicletas o el tiempo de uso. Si no se contara con el suficiente capital se recomendaría modificar el horario para así aumentar el tiempo de utilidad de las bicicletas.

\section{Referencias}

[1] Bartolomiu, John Patrick. "Cuantas calorías requiere tu cuerpo para funcionar bien". [En línea] 21 de 12 de 2015. http://www.montevideo.com.uy.

[2] Rocio. "Cómo distribuir o dividir las calorías a lo largo del día". [En línea] 14 de 01 de 2016. http://www.fuentesaludable.com/comodistribuir-o-dividir-las-caloriaslo-largo-del-dia.

Medina, 1. á. r., González, j. f. r., \& lira, j. d. v. proyecto: bicicleta generadora de energía eléctrica para alumbrado público.

Osorio, Arturo, "Generador eléctrico accionado por fuerza humana: una nueva alternativa de generación de energía", Universidad Juárez Autónoma de Tabasco, 2007 